\title{
Study on the mechanics of textile thread in woven
}

\author{
Sanovar Khamrayeva ${ }^{1 *}$, Dilfuza Kadirova $^{1}$, and Sayidvoris Rakhimkhodjayev ${ }^{1}$ \\ Tashkent Institute of Textile and Light Industry, 100100 Tashkent, Uzbekistan
}

\begin{abstract}
Specialists in the weaving industry are often interested in the question of what tension of the warp and weft threads must be set on the weaving machine to produce a particular fabric. Along with this, they are interested in the question of what is the relationship between these parameters and the structure of the fabric, how much it is necessary to increase or decrease the tension of the warp and weft threads when changing the assortment of fabric. These problems arise because the correct thread tension on weaving machines increases the productivity of equipment and labor, and also improves the quality of the fabrics produced. The current state of the mechanics of a weighty deformable flexible thread on a plane and other forms of guides do not take into account the rigidity of the threads on the friction surface, since this parameter is determined by the type and type of threads, the linear density of the threads and the elastic properties of the threads. Therefore, the article analyzes the work on the mechanics of textile yarn and studies of the tension of the yarns were carried out depending on the radius of friction, angle of friction, coefficient of friction and stiffness of the yarns.
\end{abstract}

\section{Introduction}

The question of the mechanics of a material deformable thread, constrained by a friction bond, is very widely covered in the literature. As is known, L. Euler worked a lot in this direction, where he was the first to establish the relationship between the tension of the driving and driven parts of a flexible connection sliding along the surface of a cylinder. L. Euler derived his formula for a cylinder installed horizontally, and a flexible thread wraps around the surface of this cylinder, being located on it in a plane parallel to the cylinder guide. The ends of the thread hang from the cylinder and are loaded by forces $\boldsymbol{K}$ and $\boldsymbol{K o}$ [1, 2]. The cylinder is motionless, the thread slides over its surface. The thread is weightless and inextensible and has perfect flexibility. Under these conditions, he obtained the ratio:

$$
K=K_{o} \exp (f \cdot \alpha)
$$

where, $\boldsymbol{\alpha}$ - contact angle and $\boldsymbol{f}$ - denotes the dynamic friction coefficient.

\footnotetext{
* Corresponding author: xamrayevas@mail.ru
} 
It should be noted that L. Euler considers it optional for the cylinder to be round exactly the same result for a cylinder with any radius and any shape of the guide. Euler's formula (1) gives the same thread tension $\boldsymbol{K}$ for a given wrap angle $\alpha$ and tension of the oncoming branch $\boldsymbol{K o}$, regardless of the shape of the guiding grip along which the thread is located. For example, for round cylinders with different diameters and at the same wrap angles, the tension $\boldsymbol{K}$ is the same. Undoubtedly, the thread tension cannot be the same for different forms of the cylinder guide along which the thread is located. For some cylinder shapes, it may be higher, and for others, less. In particular, the calculation of the thread tension is carried out without taking into account the friction radius.

Scientists proposed another theory, according to which the bending stiffness of a rope is considered similar to the internal resistance of a viscous fluid. The bending moment is assumed in this case in proportion to the derivative of the curvature along the arc. This theory also did not lead to satisfactory results [3-5].

Several researchers also allocate a place for the analysis of the issue of cable transmission, taking into account the rigidity and solved the problem of tension of a thread sliding according to an arbitrary law along a guide, which has a curve of arbitrary shape in its section [6-10]. However, due to the fact that when solving the problem, complex mathematical calculations were used with the use of partial differential equations, the work of A.M. Minakov was used only by a narrow circle of scientific workers and the environment of process engineers did not gain wide popularity. Moreover, an opinion arose challenging the correctness of A.P. Minakov [11].

All researchers come to the unanimous opinion that the tension of the thread after bending around a curved surface fluctuates at a high frequency, and with an increase in the speed of the thread, the average value of the tension and the magnitude of the oscillations increase sharply. Consequently, after the thread passes the guides, the tension fluctuations increase and become largely dependent on the speed and tension of the thread in the balloon.

The issues of the mechanics of a weighty deformable flexible thread on a plane and other forms of guides were considered in particular studies [11-13]. A thread of length $l$, sliding along a plane has a tension $\boldsymbol{K}_{\boldsymbol{p}} \mathrm{p}$ from its mass $\mathrm{m}$, friction radius $r$ and friction angle $\alpha$.

$$
K_{p}=m \cdot l \cdot f=m \cdot r \cdot \alpha \cdot f
$$

A thread of mass $\boldsymbol{m}$, sliding around a circle, with an arc of a girth $\boldsymbol{l}=\boldsymbol{r} \cdot \boldsymbol{\alpha}$, has a tension $\boldsymbol{K}_{\boldsymbol{c}}$ :

$$
K_{c}=\frac{2 m \cdot r \cdot f}{1+f^{2}}\left(\left(\exp (f \cdot \alpha)+\frac{1-f^{2}}{2 f} \cdot \sin \alpha-\cos \alpha\right)\right.
$$

Equations (2) and (3) determine the tension of the threads depending on the weight of the threads, the coefficient of friction and the radius of friction of the guide [11-12].

\section{Materials and Methods}

Consider the options when the guide cylinders are straight and circle. Taking into account some changes made by us in formula (2), we have [13]. The tension of the thread $\boldsymbol{K}_{\boldsymbol{p}}$ sliding along the plane has the following expression

$$
K_{p}=K_{o}+K_{H} \cdot l \cdot f
$$


where, $\boldsymbol{K}_{\boldsymbol{o}}$ - weft thread tension; $\boldsymbol{K}_{\boldsymbol{u}}$ - stiffness of the thread, depending on the type of fiber and linear density of the yarn, $\mathrm{cN} / \mathrm{mm} ; \boldsymbol{l}$ - length of thread sliding on a plane $\mathrm{mm}$; and, $\boldsymbol{f}$ coefficient of friction of the thread on the plane.

A thread of length 1, equal to the product of the friction radius $r$ by the friction angle $\alpha$, sliding along the plane has a tension $\boldsymbol{K}_{3 n}$.

$$
K_{3 n}=K_{H} \cdot l \cdot f=K_{H} \cdot r \cdot \alpha \cdot f
$$

Consequently, the tension of the thread in the runaway branch when sliding along the plane has the form:

$$
K=K_{o}+K_{3 n}
$$

A thread of length $\boldsymbol{l}$ sliding along a circle with a wrap-around arc equal to $\boldsymbol{r} \boldsymbol{\alpha}$ has a tension:

$$
K_{30}=\frac{2 K_{\mathrm{H}} \cdot r \cdot f}{1+f^{2}}\left(\exp (f \cdot \alpha)+\frac{1-f^{2}}{2 f} \cdot \sin \alpha-\cos \alpha\right)
$$

The tension of the thread in the runaway branch while sliding it around the circumference has the form:

$$
K=K_{o}+K_{3 o}
$$

When $\alpha=\pi$, the additional tension of the thread on the plane has the form [12]:

$$
K_{3 n}=K_{H} r \cdot \pi \cdot f
$$

Additional tension of the thread on the circumference of the cylinder has the form:

$$
K_{30}=\frac{2 K_{\mathrm{H}} \cdot r \cdot f}{1+f^{2}}(\exp (f \cdot \alpha)+1)
$$

\section{Results and Discussion}

Below we construct (Fig. 1) a graph of the change in the additional tension of the thread depending on the angle of friction. In calculating equations (9) and (10), it is assumed: $\boldsymbol{f}=$ 0,$2 ; \boldsymbol{\alpha}=\pi, \pi / 2, \pi / 4, \pi / 6, \pi / 8, \pi / 10$.

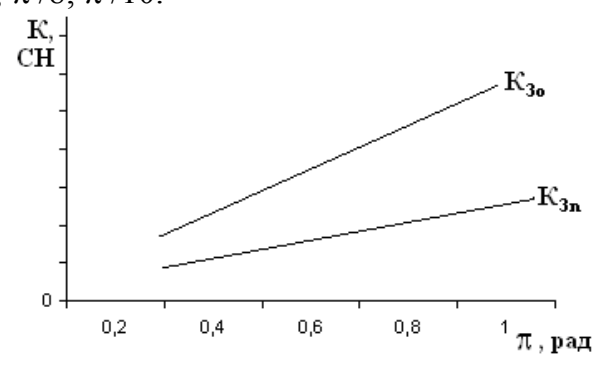

Fig. 1. Changes in the additional thread tension depending on the angle of friction. 
Also, a calculation was carried out using formulas (9) and (10) of the tension of the incoming and outgoing branches of the thread depending on the friction radius for various types of friction surfaces (plane, stationary and movable cylinder) for a thread with rigidity $\mathrm{Kn}=10 \mathrm{cN} /$ thread [13]. The calculation results are presented in Table 1.

Table 1. Results of calculating the tension of the incoming and outgoing branches of the thread at depending on the friction radius for various types of friction surfaces

\begin{tabular}{|c|c|c|c|c|c|c|c|}
\hline \multirow{2}{*}{$\begin{array}{c}\text { Friction } \\
\text { surface type }\end{array}$} & Tension of the yarn, & \multirow{2}{*}{$\begin{array}{c}\text { Coefficient } \\
\mathbf{c H} .\end{array}$} & \multicolumn{5}{|c|}{ Friction radius, $\mathbf{m m}$} \\
\cline { 5 - 9 } & of friction, $\boldsymbol{f}$ & $\mathbf{1}$ & $\mathbf{2}$ & $\mathbf{3}$ & $\mathbf{4}$ & $\mathbf{5}$ \\
\hline \multirow{2}{*}{ Plane } & Input tension, $\boldsymbol{K}_{\boldsymbol{o}}$ & 0.19 & 5.2 & 10.3 & 15.4 & 20.6 & 25.4 \\
\cline { 2 - 8 } & Output tension, $\boldsymbol{K}$ & 0.19 & 10.2 & 15.3 & 20.4 & 25.6 & 30.5 \\
\hline \multirow{2}{*}{ Fixed cylinder } & Input tension, $\boldsymbol{K}_{\boldsymbol{o}}$ & 0.19 & 13.7 & 27.3 & 41.1 & 54.6 & 59.8 \\
\hline \multirow{2}{*}{$\begin{array}{c}\text { Movable } \\
\text { cylinder }\end{array}$} & Output tension, $\boldsymbol{K}$ & 0.19 & 18.7 & 32.3 & 46.1 & 59.6 & 64.8 \\
\cline { 2 - 8 } & Input tension, $\boldsymbol{K}_{\boldsymbol{o}}$ & 0.04 & 9.6 & 19.2 & 28.8 & 38.4 & 43.4 \\
\cline { 2 - 8 } & Output tension, $\boldsymbol{K}$ & 0.04 & 14.6 & 24.2 & 33.8 & 43.4 & 53.1 \\
\hline
\end{tabular}

Determination of the coefficient of friction, we used a device developed by us to determine the coefficient of friction, which is shown in Fig. 2. For the experiment, we used guides 1 with different diameters (cylinders), thread 5 and a spring, portable strain gauge 2, located on Table 2, with the ability to move vertically [7]. The experiment is performed as follows. The thread 5 was thrown over the guiding bodies 1 , a constant weight was suspended at one end of the thread, and the tensometer lever 2 was suspended at the other end of the thread, which was evenly moved by the handle and brought the thread into a state of uniform sliding along the cylinder (yarn guide). On the scale of the tensometer, the load value at the moment of uniform sliding of the thread was noted. The readings of the tensometer correspond to the output tension of the leading branch of the tested thread $(\boldsymbol{K})$, and the value of the weight suspended from the other end of the thread corresponds to the input tension $(\boldsymbol{K o})$.

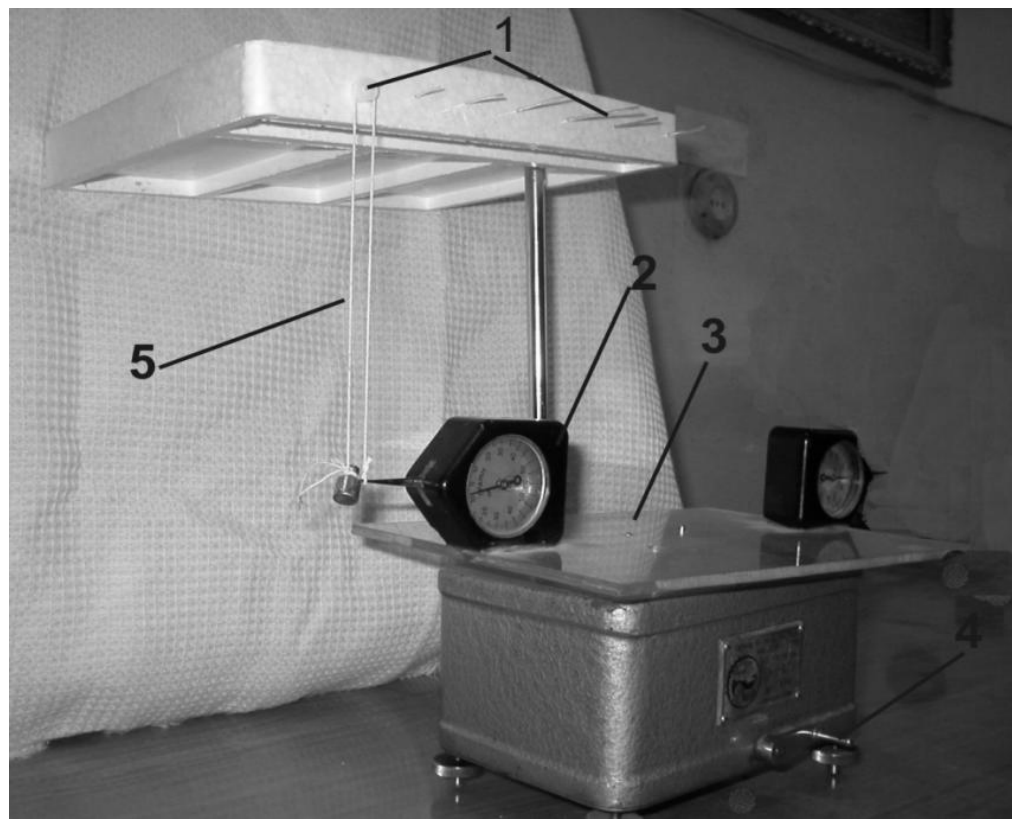

Fig. 2. Experimental setup for determining the coefficient of friction 
The experiment was carried out at a constant angle of friction equal to $180^{\circ}$, that is, $\boldsymbol{\alpha}=$ $\pi$. Table 2 shows the results of calculating the friction coefficient for various friction surfaces at $\boldsymbol{K o}=5 \mathrm{gr}$. and $\boldsymbol{\alpha}=\boldsymbol{\pi}$, linear density of the yarn 320 tex.

Table 2. Results of calculating the coefficient of friction for various friction surfaces

\begin{tabular}{|c|c|c|c|}
\hline Surface friction & $\begin{array}{c}\text { Condition frictional } \\
\text { couples }\end{array}$ & $\begin{array}{c}\text { Indication } \\
\text { tensometer, g }\end{array}$ & $\begin{array}{c}\text { Coefficient } \\
\text { friction of the } \\
\text { thread on the } \\
\text { surface } \\
\end{array}$ \\
\hline \multirow{2}{*}{$\begin{array}{c}\text { Rotating } \\
\text { cylinder, } \\
\text { plastic-metal surface }\end{array}$} & uniform movement & 5.5 & 0.04 \\
\hline & $\begin{array}{l}\text { at the beginning of the } \\
\text { movement, at rest }\end{array}$ & 6.0 & 0.07 \\
\hline \multirow[b]{2}{*}{$\begin{array}{c}\text { Plane, stationary } \\
\text { cylinder surface-metal }\end{array}$} & uniform movement & 8.0 & 0.19 \\
\hline & $\begin{array}{l}\text { at the beginning of the } \\
\text { movement, at rest }\end{array}$ & 8.7 & 0.23 \\
\hline \multirow{2}{*}{$\begin{array}{l}\text { Plane, stationary } \\
\text { cylinder surface - } \\
\text { plastic }\end{array}$} & uniform movement & 10.0 & 0.28 \\
\hline & $\begin{array}{l}\text { at the beginning of the } \\
\text { movement, at rest }\end{array}$ & 15.0 & 0.45 \\
\hline \multirow{2}{*}{$\begin{array}{l}\text { Plane, stationary } \\
\text { cylinder surface- } \\
\text { rubber }\end{array}$} & uniform movement & 20.0 & 0.56 \\
\hline & $\begin{array}{l}\text { at the beginning of the } \\
\text { movement, at rest }\end{array}$ & 25.0 & 0.66 \\
\hline
\end{tabular}

Analysis of Equations (2) and (3) determine the tension of the threads depending on the weight of the threads, the coefficient of friction and the radius of friction of the guide. Moreover, the mass of threads does not take into account the type and type of threads, the linear density of the threads and the elastic properties of the threads. For example, the mass of $1 \mathrm{~kg}$ of cotton thread and $1 \mathrm{~kg}$ of silk thread have the same values, in particular, there are no indicators of the properties of the threads, the linear density of the threads, the elasticity of the threads, deformation, etc. Consequently, the mass of threads does not cover the quantitative and qualitative characteristics of the threads and their properties. Our proposed formulas (5) and (7) take into account the stiffness of the threads on the friction surface, since this parameter is determined by the type and type of threads, the linear density of the threads and the elastic properties of the threads. Therefore, it is advisable to study the tension of the threads on the basis of taking into account the coefficient of rigidity of the threads. Additional tension of the thread on the plane and on the circumference of the cylinder at $\alpha=\pi$, (Fig.1) confirms that, depending on the type of guide (plane or cylinder) through which the thread is thrown, we obtain at the same stiffness of the thread, the friction radius, the coefficient of friction and the initial tension (on the oncoming branch of the thread) are different values of the thread tension. For a thread sliding along the plane, the tension of the thread is less than for a thread sliding around the circumference.

Analysis of Table 1 shows that with an increase in the friction radius for various types of friction surfaces, the tension of the incoming and outgoing branches of the thread increases. From Table 2 it follows that at the beginning of sliding of the thread, the surface corresponding to the coefficient of friction at rest in all variants of the experiment is greater than the values of the coefficient of friction in motion corresponding to the state of the friction pair (thread-surface) of uniform movement.

\section{Conclusions}

An experimental setup has been developed for determining the friction coefficient and a method for determining the friction coefficient of a weft depending on the shape, size and state of friction surfaces. An increase in the thread friction radius leads to an increase in the 
thread tension. The coefficient of friction at rest is greater than the coefficient of friction in motion in all variants of rubbing surfaces. The equations for the tension of the threads sliding along the plane, along the circumference of the stationary and movable cylinders, taking into account the rigidity of the thread, the radius of friction, the angle of friction and the coefficient of friction are obtained.

\section{References}

1. Kh. Yu. Rasulov, S. S. Raximxodjayev, International Journal of Advanced Research in Science, Engineering and technology 5(10), 7001-7005 (2018)

2. S. S. Raximxodjayev, D. Kadirova, Theory of wool processing, 212 (Adabiyot uchkunlari Press, Tashkent, 2018)

3. S. Xamrayeva, D. Kadirova, S. Raximxodjayev, J. Academy 10(61), 12 (2020)

4. S. Raximxodjayev, D. Kadirova, Problems of textile 4, 11 (2002)

5. D. Kadirova, A. Daminov, S. Rahimhodjaev, Technology of production of technical belts and the study of their properties, 549-552 (2019)

6. D. Kadirova, International Journal of Advanced Research in Science, Engineering 11(3), 18 (2018)

7. S. Raximxodjayev, D. N. Kadirova, Problems of textile 4, 17 (2013)

8. D. Kodirova, S. Raximxodjayev, Problems of textile 3, 11-14 (2002)

9. G. Miroshnichenko, Mechanical engineering, 458-468 (1972)

10. E. Zikrieev, Primary processing of raw cotton, 400 (Mekhnat Press, Tashkent, 1999)

11. I. Maksudov, A. Nuraliev, Reference book of the primary processing of cotton, 395 (Mekhnat Press, Tashkent, 1995)

12. I. Shin, A. Juraev, Problems of textiles 1, 7-11 (2009)

13. I. Shin, Hardening technologies and coatings 10, 24-26 (2010) 